\title{
APLICACIÓN DE MÉTODOS ANALÍTICOS PARA EL CÁLCULO DE ABATIMIENTOS EN ACUÍFEROS CONFINADOS
}

\author{
Asdrúbal Vargas \\ Instituto Costarricense de Electricidad \\ Centro Servicio Exploración Subterránea \\ E-Mail: avargass@ice.go.cr
}

\begin{abstract}
Two analytical methods are presented to calculate the number and discharge of pumping wells for the drawdown of very high piezometric levels. These methods were applied to the confined aquifer in the Angostura Hydroelectric Project and facilitate a battery of six wells with a mean discharge of $27 \mathrm{l} / \mathrm{s}$. The well design includes a well screen of $24 \mathrm{~m}$ with a diameter of $0,1 \mathrm{~m}$ located to a depth between 48 to $52 \mathrm{~m}$. These analytical methods can help to calculate the costs of wells in dewatering projects and is very useful to prepare cost budgets.

The method of big diameter well estimates the number of wells required and the method of discharge can calculate the effective discharge of each well. Both methods operate in electronic sheets and make the calculations easy.
\end{abstract}

RESUMEN: En este trabajo se presentan dos metodologías de tipo matemático analítico con el fin de obtener el número de pozos y el caudal de cada uno de ellos para abatir el nivel piezométrico de acuíferos confinados. Estos métodos se aplicaron al caso de un acuífero confinado que se encuentra debajo del vertedero principal del proyecto hidroeléctrico Angostura. Por medio de estos métodos se obtiene una batería de seis pozos, distribuidos alrededor del vertedero con caudales de explotación cercanos a los 27 1/s. El diseño geomecánico de los pozos comprende una rejilla de $24 \mathrm{~m}$ de longitud con un diámetro de $0,1 \mathrm{~m}$ (4 pulgadas) ubicada a una profundidad que varía de 48 a $52 \mathrm{~m}$.

Los métodos analíticos han demostrado ser válidos para obtener una aproximación del problema planteado. El método del gran pozo permite determinar el número de pozos y el método del caudal permite determinar el caudal adecuado de cada pozo. Estos métodos se pueden aplicar para determinar el número de pozos de bombeo necesarios para abatir el nivel piezométrico en excavaciones o minas a cielo abierto y permite elaborar presupuestos de costos dentro de los planes de construcción de proyectos hidroeléctricos o aquellos donde se contemplen este tipo de obras civiles.

\section{INTRODUCCIÓN}

En ocasiones, durante la construcción de obras civiles (vertederos, excavaciones) surge la necesidad de abatir el nivel piezométrico del agua subterránea para evitar efectos geotécnicos indeseables en la base de la obra a construir. So- bre todo es necesario la implementación de dichas acciones cuando se requiere realizar una excavación para la ubicación de un vertedero o la fundación de la casa de máquinas como parte de un proyecto hidroeléctrico. El cálculo del número de pozos necesario para abatir el nivel piezométrico hasta un valor previamente calculado a 
partir del análisis de fuerzas, es una tarea que se puede lograr por medio de métodos matemáticos de tipo analítico.

Los métodos analíticos tienen la ventaja de incorporar expresiones matemáticas sencillas derivadas de la hidráulica de pozos y permiten realizar aproximaciones utilizando una serie de condiciones de isotropía y homogeneidad del medio acuífero. En este caso se utilizarán dos métodos analíticos para el cálculo del número de pozos necesario y el caudal adecuado de cada pozo para abatir el nivel piezométrico y se aplicará al caso del vertedero principal del proyecto hidroeléctrico Angostura.

El proyecto hidroeléctrico Angostura está concebido para la generación de 177 MW de energía utilizando el agua transportada principalmente por el río Reventazón, y consta de embalse, presa, vertedero, túnel y casa de máquinas. La construcción de algunas de las obras mencionadas han requerido de acciones complementarias para disminuir la influencia del agua subterránea. Por ejemplo, la excavación del vertedero principal requiere la disminución de la presión del agua en el acuífero para evitar levantamientos y surgencia de agua por medio de grietas o fallas. Según los análisis geotécnicos se requiere abatir el nivel de agua en un valor que oscila de 16 a $19 \mathrm{~m}$.

El objetivo de este trabajo es el de aplicar dos métodos matemáticos de tipo analítico para obtener el diseño más adecuado de la batería de pozos que se requiere para abatir el nivel de agua subterránea en el acuífero inferior que se encuentra en el sector de la presa y los vertederos.

En el año de 1994 se inició la construcción del proyecto, el cual se ubica a $7 \mathrm{~km}$ al este de la ciudad de Turrialba (Fig. 1). Estudios hidrogeológicos en el sitio han sido llevados a cabo por Vargas (1997) y Vargas \& Cervantes (2000), donde se describen las condiciones y las características de los principales acuíferos.

La metodología empleada en este trabajo ha sido dividida en varias fases, incluyendo la búsqueda de información referente a metodologías de análisis de abatimientos de niveles de agua subterránea por medio de múltiples pozos alrededor de excavaciones. Herth \& Arndts (1994), y Custodio \& Llamas (1983) presentan

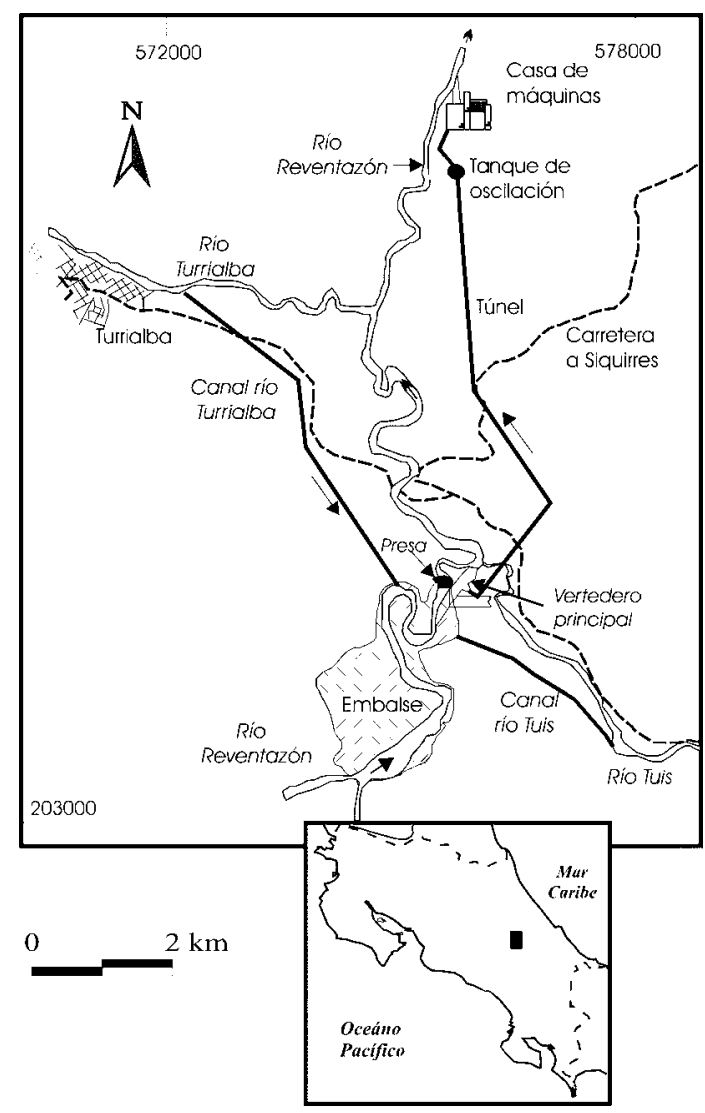

Fig. 1: Ubicación del proyecto hidroeléctrico Angostura y de las obras principales.

un método que utiliza la expresión de Jacob, modificada para el caso de múltiples pozos. Una vez identificada la metodología de análisis se procedió a recopilar datos e introducirlos en las expresiones matemáticas correspondientes para obtener el número adecuado de pozos o el caudal requerido según el caso.

\section{CONDICIONES HIDROGEOLÓGICAS}

Los perfiles de perforación obtenidos del área de la presa y de los vertederos permiten diferenciar 4 horizontes aluvionales, los cuales están separados por flujos laháricos. El horizonte aluvional más profundo se puede diferenciar de los otros tres por medio de la elevación del límite inferior o superior. 
A partir de la información extraída de las perforaciones, se deduce que el acuífero inferior presenta la mayor extensión y el agua se encuentra a presiones superiores a $6 \mathrm{~kg} / \mathrm{cm}^{2}$. Se extiende a partir de la presa en dirección este, pasando por el río Tuis y parcialmente por el valle del río Reventazón con un ancho aproximado de $200 \mathrm{~m}$. Se supone, además, una extensión de este horizonte en dirección norte, la cual debe ser comprobada. En su conjunto se considera este horizonte aluvional como un sistema antiguo del río Reventazón. El espesor de este acuífero alcanza entre 10 y $27,5 \mathrm{~m}$. El límite superior está a la cota de 500 m.s.n.m en el área de la presa y en el sector del embalse y el límite inferior entre 475480 m.s.n.m. Los valores de transmisividad son de alrededor de 500-600 $\mathrm{m}^{2} /$ día y los coeficientes de almacenamiento alrededor de $10^{-4}$ (Vargas $\&$ Cervantes, 2000). Conociendo las características hidrogeológicas básicas del acuífero se procederá a realizar un análisis utilizando dos métodos analíticos para determinar por un lado el número adecuado de pozos y por otro el caudal de extracción de cada pozo.

\section{MÉTODO DEL GRAN POZO}

Esta metodología fue desarrollada por un grupo de investigadores holandeses y presentada en Herth \& Arndts (1994). Consiste en calcular el caudal de un pozo que tiene las dimensiones de la excavación y el caudal de un pozo particular considerando la elevación del nivel piezométrico en el sitio de la excavación. Posteriormente el caudal del pozo de mayor dimensión se divide por el caudal del pozo sencillo y se obtiene el número de pozos necesario para alcanzar el abatimiento requerido en los pozos. Más adelante se estima el caudal requerido en cada pozo individual para alcanzar el abatimiento requerido en los pozos.

Como primera etapa se realiza el cálculo del caudal de extracción de un pozo con dimensiones similares a la excavación.

$$
Q=\frac{\pi \cdot k \bullet m \bullet s \bullet 2}{2,3 \bullet\left(\log R-\log A_{R E}\right)}
$$

Donde:

k: $\quad$ conductividad hidráulica del acuífero $(\mathrm{m} / \mathrm{s})$

$\mathrm{s}$ : abatimiento necesario dentro de la excavación (m)

$\mathrm{R}: \quad$ radio de influencia del pozo $(\mathrm{m})$

$\mathrm{A}_{\mathrm{RE}}:$ radio interno del pozo $(\mathrm{m})$

El radio de influencia se puede estimar según la expresión de Sichardt (en Herth \& Arndts, 1994).

$$
R=3000 \bullet s \cdot \sqrt{k}
$$

El término $A_{R E}$ se puede estimar por medio de las dimensiones del largo y ancho de la excavación.

Donde:

$$
A_{\mathrm{RE}}=\eta^{*} \mathrm{~b}
$$

$\eta=$ valor adimensional que se obtiene a partir de las dimensiones de la excavación representadas en el gráfico a/b versus $\mathrm{h}$ (Herth \& Arndts, 1994, pág. 87)

a: lado largo de la excavación

b: lado corto de la excavación $\mathrm{m}=\mathrm{a} / \mathrm{b}$

Por su parte el caudal del pozo particular se obtiene por medio de la siguiente expresión:

$$
q=\pi \cdot \phi \cdot h^{\prime} \cdot \frac{\sqrt{k}}{15} \quad\left(\frac{m^{3}}{s}\right)
$$

Donde:

q: $\quad$ caudal de cada pozo $\left(\mathrm{m}^{3} / \mathrm{s}\right)$

$\phi(\mathrm{m})$ : diámetro del pozo de bombeo

h ' (m): columna de agua desde la base del acuífero hasta el nivel piezométrico máximo

k: conductividad hidráulica del acuífero $(\mathrm{m} / \mathrm{s})$

El número de pozos aproximado, necesario para abatir el nivel del agua en la excavación, se calcula dividiendo el caudal mayor por el caudal de cada pozo de extracción:

$$
n=\frac{Q}{q}
$$


Para el caso del proyecto hidroeléctrico Angostura las dimensiones de los factores se presentan en el cuadro 1. En el procedimiento anterior se ha calculado el número de pozos teniendo en cuenta el abatimiento requerido debajo de la excavación. Sin embargo, con el fin de obtener el caudal que asegure el abatimiento aún en los extremos de la excavación, es necesario también realizar el cálculo del caudal considerando un punto ubicado en un extremo para lo cual se utiliza generalmente la distancia desde un punto ubicado en una de las esquinas a los pozos de alivio y obteniendo el promedio de los logaritmos. A continuación se procede a calcular la distancia promedio de manera logarítmica por medio de la expresión.

$$
X_{\text {med }}=\frac{1}{n} \cdot \sum \log x
$$

Donde :

$$
\begin{aligned}
& \mathrm{n}=\quad \text { número de pozos de extracción obteni- } \\
& \text { dos en la primera fase } \\
& \mathrm{x}=\quad \text { distancia desde el punto escogido hasta } \\
& \text { los pozos de extracción }
\end{aligned}
$$

\begin{tabular}{|c|c|}
\hline Parámetro & Valor $(\mathrm{m})$ \\
\hline Espesor acuífero promedio (m) & 27,0 \\
\hline Elevación de nivel sobre techo acuífero $(\mathrm{m})$ & 78,0 \\
\hline H Columna agua $(\mathrm{m})$ & $(27+78) \quad 105,0$ \\
\hline $\mathrm{k}(\mathrm{m} / \mathrm{d})$ & 24,0 \\
\hline $\mathrm{k}(\mathrm{m} / \mathrm{s})$ & 0,00027 \\
\hline $\mathrm{R}$ influencia (m) según Sichardt & 850,0 \\
\hline a Lado largo excavación(m) & 170,0 \\
\hline b Lado corto excavación (m) & 100,0 \\
\hline $\mathrm{m}=\mathrm{a} / \mathrm{b}(-)$ & 1,7 \\
\hline n (-) Obtenido de Herndt \& Arndts (1994) & 0,75 \\
\hline $\mathrm{A}_{\mathrm{Re}} \cdot$ Pozo $=$ excavación $(\mathrm{m})$ & 75,0 \\
\hline $\mathrm{d}$ Diámetro pozo indiv. (m) & 0,25 \\
\hline $\mathrm{H}^{\prime} \quad$ Elevación superficie del agua (m) & 75,0 \\
\hline Abatimiento necesario en excavación (m) & 17,0 \\
\hline Caudal pozo excavación $\left(\mathrm{m}^{3} / \mathrm{s}\right)$ & 0,32 \\
\hline Caudal máximo $\left(\mathrm{m}^{3} / \mathrm{s}\right)$ & 0,35 \\
\hline Caudal máximo (1/s) & 350,0 \\
\hline q Caudal cada pozo individual $\left(\mathrm{m}^{3} / \mathrm{s}\right)$ & 0,064 \\
\hline q Caudal cada pozo individual(l/s) & 64,0 \\
\hline Numero pozos bombeo & 6 \\
\hline
\end{tabular}

Cuadro 1

Parámetros para el cálculo del número de pozos
$\mathrm{X}_{\text {med }}=$ distribución óptima de los pozos considerando la distancia desde los pozos hasta un punto en la esquina de la excavación.

Para el caso en estudio se utilizan los seis pozos ubicados en las bermas del vertedor. La distancia desde el punto de interés (B) a los pozos varía de 71 a $134 \mathrm{~m}$ (Cuadro 2). Ahora se procede a calcular el caudal del pozo de gran diámetro usando el valor medio de los logaritmos de las distancias (Xmed) dentro de la expresión E1, obteniéndose un valor de $0,36 \mathrm{~m}^{3} / \mathrm{s}$.

$$
Q\left(\frac{m^{3}}{s}\right)=\frac{\pi \cdot 0,00027 \cdot 27 \cdot 17 \cdot 2}{2,3 \cdot(\log 850-1,99)}=0,36
$$

Teniendo en cuenta el número de pozos obtenidos en la primera fase se obtiene a continuación el caudal de cada pozo, dividiendo el caudal real $(\mathrm{Q})$ por el número de pozos; resulta un valor de $0,06 \mathrm{~m}^{3} / \mathrm{s}(60 \mathrm{l} / \mathrm{s})$.

$$
q=\frac{Q}{n}
$$

Adicionalmente se requiere calcular el abatimiento que ocurrirá en los pozos de bombeo, que se obtiene por medio de la siguiente expresión:

Cuadro 2

Calculo de caudal real considerando punto externo

\begin{tabular}{lcc}
\hline \multicolumn{1}{c}{ No. Pozo } & Dist. x a B & Log x \\
\hline 1 & 71,40 & 1,85 \\
2 & 102,00 & 2,01 \\
3 & 126,50 & 2,10 \\
4 & 134,70 & 2,13 \\
5 & 100,00 & 2,00 \\
6 & 71,40 & 1,85 \\
Suma Log x & & 11,95 \\
$1 /$ numero pozos & 0,167 & \\
$1 / \mathrm{n} *$ Suma LOG & 1,99 & \\
Caudal real (m $3 / \mathrm{s})$ & 0,371 & \\
Caudal real $(1 / \mathrm{s})$ & 371,25 & \\
Caudal por pozo (m $3 / \mathrm{s})$ & 0,062 & \\
Diferencia de niveles $(\mathrm{m})$ & 11,8 & \\
Elevación real nivel agua $(\mathrm{m})$ & 70,9 & \\
Caudal real final pozo $\left(\mathrm{m}^{3} / \mathrm{s}\right)$ & 0,062 & \\
\hline
\end{tabular}




$$
s_{E B}=\frac{1,5 \bullet q \cdot 2,3 \bullet(\log b-\log r)}{3,1415 \cdot k \cdot 2 \cdot m}
$$

Donde:

q: caudal de cada pozo $\left(\mathrm{m}^{3} / \mathrm{s}\right)$

$\mathrm{k}$ : conductividad hidráulica del acuífero $(\mathrm{m} / \mathrm{s})$

b: mitad de la distancia media entre dos pozos, que para este caso se ha calculado dividiendo el largo de la excavación entre el número de pozos y que corresponde a $57 \mathrm{~m}$

$\mathrm{r}$ : radio del pozo $(\mathrm{m})$

$\mathrm{m}$ : espesor del acuífero $(\mathrm{m})$

$$
s_{E B}=\frac{3,45 \bullet 0,06 \bullet(\log 57,0-\log 0,12)}{3,1415 \bullet 0,00027 \cdot 2 \cdot 27}=12,1 \mathrm{~m}
$$

Por último es necesario calcular la altura de la rejilla ( $\left.h^{\prime}\right)$ que se requiere para que cada pozo extraiga un caudal " $q$ ". Con esto se obtiene un valor de $24,2 \mathrm{~m}$.

$$
h^{\prime}=\frac{15 \cdot q}{2 \cdot r \cdot 3,1415 \cdot \sqrt{k}}=24,2
$$

Es recomendable verificar que se cumple la condición $\mathrm{h}^{\prime} \leq \mathrm{h}-\mathrm{S}_{\mathrm{EB}}$, donde $\mathrm{h}$ es la elevación desde la base del acuífero hasta el nivel de abatimiento debajo de la excavación (Fig. 2). En este caso $24,2 \leq 84,1 \mathrm{~m}$. En conclusión, con este método se llega a demostrar que el número de pozos estimado que se ubicarían alrededor de la excavación del vertedero es capaz de alcanzar el abatimiento esperado de $17 \mathrm{~m}$ debajo de la excavación que permitiría disminuir la presión del agua subterránea.

\section{MÉTODO DE LOS CAUDALES DE EXTRACCIÓN}

Otro método por medio del cual se obtiene el caudal de cada pozo, necesario para abatir el nivel piezométrico en una excavación, consiste en resolver una matriz compuesta de ecuaciones no lineales definidas por el número de pozos de la batería de extracción por medio de un algoritmo de optimización que hoy en día se puede encontrar en varios programas de manejo de hojas electrónicas. Dentro de estas ecuaciones se cuenta
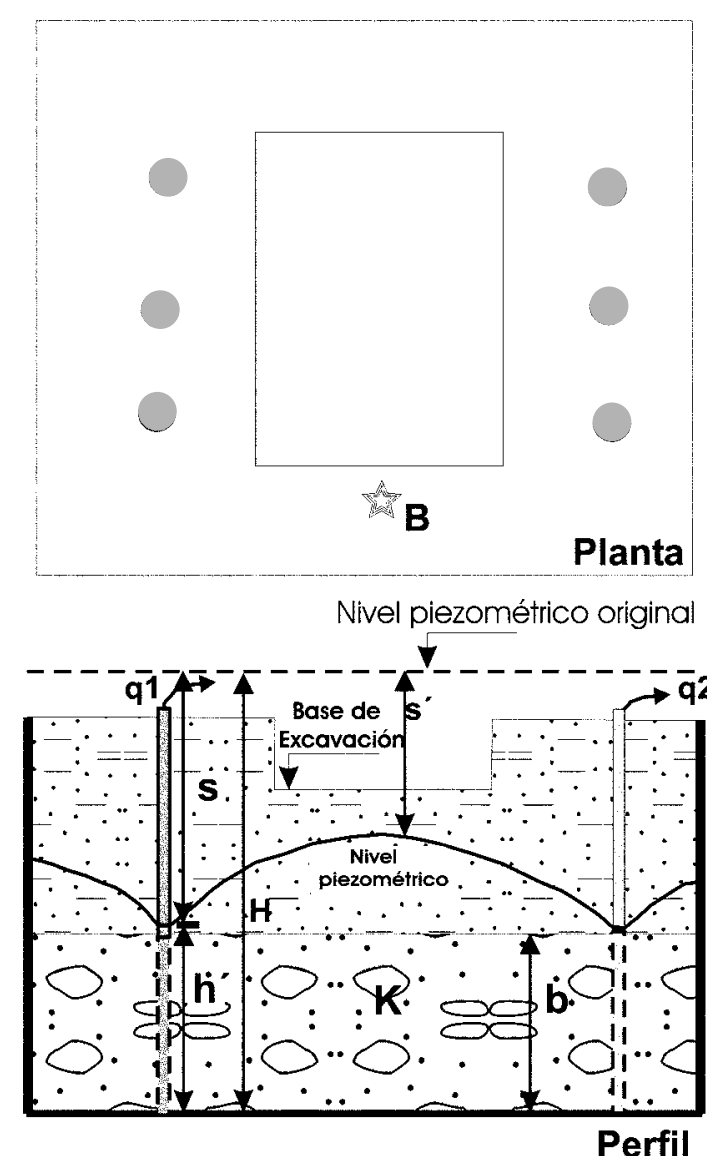

Fig. 2: Esquema de distribución de los pozos alrededor de la excavación y nomenclatura de los parámetros utilizados en el método del gran pozo.

con "n" incógnitas, a saber el caudal de extracción de "n" pozos de bombeo.

Este método tiene una ventaja sobre el método anterior, ya que es posible calcular el caudal de cada pozo tomando en cuenta las condiciones tanto en el acuífero como en el pozo; es decir, toma en cuenta las pérdidas por fricción dentro del acuífero y en el pozo, lo cual aumenta la certidumbre sobre las estimaciones. Considerando que el acuífero se encuentra en régimen permanente y de tipo confinado, el abatimiento en el pozo se obtiene a partir de la expresión de Custodio \& Llamas (1983, p. 685), donde el descenso en uno de los pozos de bombeo será el que produce el propio bombeo más el producido por el conjunto de los restantes pozos. 


$$
\mathrm{s}_{\mathrm{pi}}=\left(\Sigma\left(\mathrm{Q}_{\mathrm{i}} \mathrm{Z}(\mathrm{r}, \mathrm{t})\right)\right.
$$

siendo $\mathrm{r}$ la distancia de cada pozo al pozo i y $\mathrm{Q}$ $Z$ la ecuación del descenso provocado por el pozo i. Por su parte la ecuación del descenso de cada pozo corresponde a:

$$
s_{i}=\left(\frac{1}{2 \cdot \pi \cdot T}\right) \cdot \sum\left(Q_{i} \cdot \operatorname{Ln}\left(\frac{R}{r_{i}}\right)\right)
$$

Donde

$\mathrm{s}_{\mathrm{i}}$ : abatimiento máximo en el pozo i $(\mathrm{m})$

$\mathrm{T}$ : $\operatorname{transmisividad~}\left(\mathrm{m}^{2} / \mathrm{d}\right)$

$\mathrm{Q}_{\mathrm{i}}$ : caudal de cada pozo de bombeo en el campo de pozos $\left(\mathrm{m}^{3} / \mathrm{d}\right)$

$\mathrm{R}$ : radio de influencia de cada pozo $(\mathrm{m})$

$r_{i}$ : distancia del pozo i a cada pozo del campo de pozos $(\mathrm{m})$. La distancia $\mathrm{r}_{\mathrm{i}}$ del pozo i al mismo pozo será tomado como el radio del pozo

Tomando en cuenta las pérdidas por fricción, la ecuación resultante será:

$$
s_{i}=\frac{1}{2 \cdot \pi \cdot T} \cdot \sum\left(Q_{i} \cdot \operatorname{Ln} \frac{R}{r_{i}}\right)+C \cdot Q^{2}
$$

donde $\mathrm{C}$ es un factor que depende de las pérdidas de fricción en el pozo y se puede calcular de acuerdo a expresiones matemáticas que toman en cuenta las características de las tuberías y elementos de construcción del pozo. Lo primero que se hará, por lo tanto, es calcular las pérdidas totales en la estructura del pozo.

\section{Pérdidas por fricción en el sistema hidráulico}

La conducción del agua a través de tubería origina una fricción que provoca pérdidas de carga que disminuyen el nivel dinámico. Debido a que estas pérdidas por fricción alcanzan un valor en ocasiones superior a las pérdidas en el acuífero es necesario calcularlas.

\section{Pérdidas de carga por entrada en la rejilla (Per)}

Las pérdidas de carga por fricción a la entrada de la rejilla se obtienen según la siguiente expresión, expresándose las unidades como $\mathrm{seg}^{2} / \mathrm{m}^{5}$.

$$
P_{e r}=k \cdot\left(\frac{Q^{2}}{2 \cdot g \cdot A n^{2}}\right)
$$

Donde:

$$
k=1,45-\left(0,45 \cdot\left[\frac{A n}{A b}\right]-\left[\frac{A n}{A b}\right]^{2}\right)
$$

An: área neta de paso entre rejillas expresada en $\mathrm{m}^{2}$, la cual está en función de la abertura entre los hilos de la rejilla y depende del tipo de rejilla, obteniéndose el área por metro de rejilla tipo Jhonson de la referencia Driscoll (1986).

Ab: área bruta de la estructura de la rejilla expresada en $\mathrm{m}^{2}$, la cual se calcula tomando en cuenta el ancho (circunferencia) de la rejilla y multiplicando por el largo de la misma.

Q: Caudal de entrada $\left(\mathrm{m}^{3} / \mathrm{s}\right)$

g: fuerza de la gravedad $\left(\mathrm{m} / \mathrm{s}^{2}\right)$

Los valores utilizados para calcular el valor de pérdidas de carga por fricción se muestran en el cuadro 3, así como el porcentaje del total, que se obtiene como una proporción de las pérdidas totales. El porcentaje de pérdidas de carga por entrada a la rejilla es muy bajo y es uno de los factores que menor influencia tiene, comparado con las pérdidas en otros puntos del pozo como se verá a continuación.

\section{Pérdidas de carga dentro de la rejilla (Pdr)}

Las pérdidas de carga por fricción dentro de la rejilla se obtiene según la siguiente expresión.

\section{Cuadro 3}

Pérdidas por fricción por entrada a la rejilla

\begin{tabular}{lc}
\hline Parámetro & Valor (m) \\
\hline Longitud rejilla (m) & 24 \\
Ancho rejilla (m) & 0,31 \\
Area neta de paso (m2) & 0,7567 \\
Area bruta de rejilla (m2) & 7,44 \\
K & 1,39 \\
Pérdida por entrada rejilla (seg2/m5) & 0,12 \\
Porcentaje del total $(\%)$ & 0,00366 \\
\hline
\end{tabular}




$$
P_{d r}=f_{o}\left(\frac{L}{D^{5}}\right) \cdot\left(\frac{8}{[\pi]^{2} \cdot g}\right) \cdot Q^{2}
$$

Donde:

fo : factor de fricción y corresponde a un tercio del valor del factor $\mathrm{f}$, que se calcula según la expresión.

$$
f=\frac{1}{4\left(\operatorname { L o g } ^ { 2 } \left[\frac{3,71}{\left.\left\{\frac{\varepsilon}{D}\right]\right)}\right.\right.}
$$

donde $\varepsilon$ equivale a la rugosidad de la rejilla, que tiene un valor de $2 \mathrm{~mm}$.

L: longitud de la rejilla $(\mathrm{m})$

D: diámetro de la tubería $(\mathrm{m})$

g: fuerza de la gravedad $\left(9,8 \mathrm{~m} / \mathrm{s}^{2}\right)$

Q: caudal de extracción $\left(\mathrm{m}^{3} / \mathrm{s}\right)$

El valor de cada parámetro para dicha expresión, se muestra en el cuadro 4, notándose que el porcentaje de pérdidas con respecto al total es bastante elevado. En este caso se ha considerado una rugosidad de $2 \mathrm{~mm}$ para rejilla de hierro galvanizado.

Cuadro 4

Pérdidas de carga dentro de la rejilla

\begin{tabular}{lc}
\hline Parámetro & Valor (m) \\
\hline Diámetro rejilla (m) & 0,10 \\
Longitud rejilla (m) & 24,00 \\
Rugosidad (E) (m) & 0,002 \\
F & 0,049 \\
Fo & 0,016 \\
Pérdida dentro rejilla $\left(\mathrm{seg}^{2} / \mathrm{m}^{5}\right)$ & 3215,16 \\
Porcentaje del total $(\%)$ & 94,78 \\
\hline
\end{tabular}

\section{Pérdidas de carga por entrada a la tubería de conducción (Petc)}

Luego del movimiento del agua dentro de la rejilla, esta entra a la tubería de conducción, donde se presentan cambios de diámetro, por lo que también ocurren pérdidas de carga, que se calculan según la siguiente expresión.

$$
P_{e t c}=\left(\frac{k}{D^{4}}\right) \cdot\left(\frac{8}{\left\{\pi^{2} \cdot g\right\}}\right) \cdot Q^{2}
$$

Donde el factor K se toma como 0,5 (Sotelo, 1993). Para este tramo del pozo el diámetro ha variado a $0,24 \mathrm{~m}$ (Cuadro 5), debido a que el diseño del pozo incluye un diámetro de perforación mayor desde la superficie hasta $48 \mathrm{~m}$ de profundidad y luego se cambia a un diámetro menor para la rejilla (Fig. 3). Como se nota de la expresión de cálculo las pérdidas están en función del cambio del diámetro de la tubería.

Cuadro 5

Pérdidas de carga por entrada a la tubería

\begin{tabular}{lc}
\hline Parámetro & Valor (m) \\
\hline $\mathrm{K}$ & 0,50 \\
Diámetro tubería $(\mathrm{m})$ & 0,24 \\
Pérdida entrada a tubería $\left(\mathrm{seg}^{2} / \mathrm{m}^{5}\right)$ & 12,47 \\
Porcentaje del total $(\%)$ & 0,37 \\
\hline
\end{tabular}

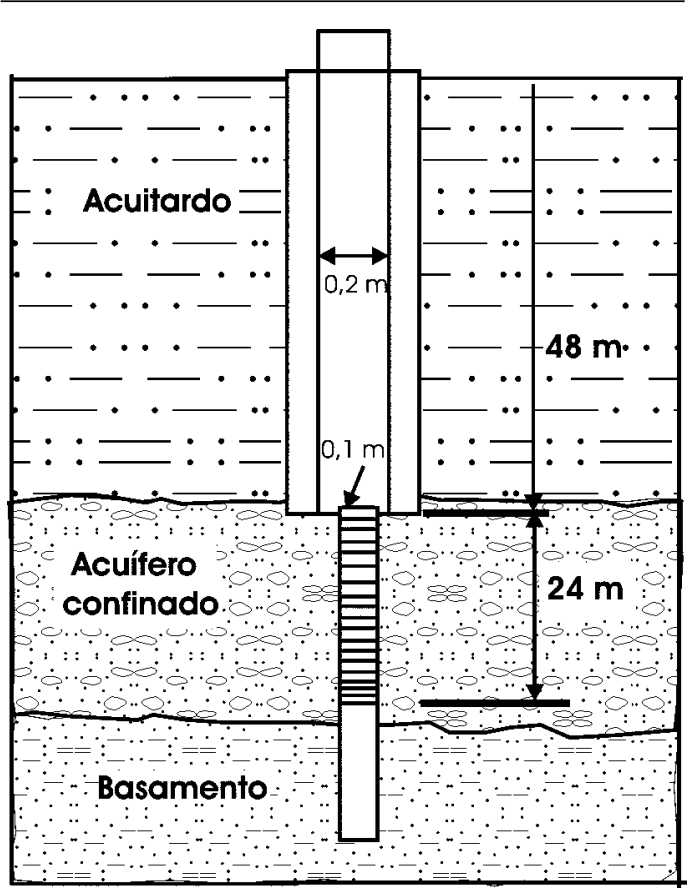

Fig. 3: Diseño geomecánico de un pozo de extracción que se utilizaría en la batería de pozos, mostrando los puntos de pérdidas de velocidad (energía) del agua por fricción. 


\section{Pérdidas dentro de la tubería de conducción (Pdt)}

El agua que fluye dentro de la tubería se encuentra en rozamiento con las paredes y se produce una pérdida de carga que se calcula según la siguiente fórmula.

$$
q=\frac{Q}{n}
$$

Donde $\mathrm{f}=$ 0,026 (Sotelo,1993; p. 284) y depende del tipo de tubería y su rugosidad. L y D corresponden a la longitud y al diámetro de la tubería (Cuadro 6). Las pérdidas obtenidas se encuentran en segundo lugar con respecto al total, sin embargo debido a un diámetro mayor dicho valor es bajo.

$$
P_{c d}=\left(\frac{K}{D^{4}}\right) \cdot\left[\frac{8}{\left\{\pi^{2} \bullet g\right\}}\right] \cdot Q^{2}
$$

Cuadro 6

Pérdidas de carga dentro de la tubería

\begin{tabular}{lc}
\hline Parámetro & Valor $(\mathrm{m})$ \\
\hline Diámetro tubería $(\mathrm{m})$ & 0,24 \\
Longitud tubería $(\mathrm{m})$ & 48 \\
$\mathrm{f}$ & 0,026 \\
Pérdida dentro tubería $\left(\mathrm{seg}^{2} / \mathrm{m}^{5}\right)$ & 129,64 \\
Porcentaje del total $(\%)$ & 3,82 \\
\hline
\end{tabular}

\section{Pérdidas de carga por cambio de dirección de la tubería de conducción (Pcd)}

El agua extraída de la formación es obligada a desviarse por medio de un codo con un ángulo de $90^{\circ}$ lo que provoca una pérdida de carga, estimada por la siguiente expresión.

El valor de $\mathrm{K}$ depende de un factor $\mathrm{Cc} \mathrm{y}$ del ángulo del codo $(\Theta)$, de tal manera que

$$
K=C_{c} \frac{\Theta}{90^{0}}
$$

sin embargo, para un codo de radio grande, $\mathrm{K}$ $=0,4$ se obtiene de tablas (Sotelo,1993; p. 306). Los valores de los parámetros involucrados en la fórmula se presentan en el cuadro 7, obteniéndose un valor relativamente bajo por pérdidas de carga por concepto de cambio de dirección.
Cuadro 7

Pérdidas de carga por cambio de dirección

\begin{tabular}{lc}
\hline Parámetro & Valor $(\mathrm{m})$ \\
\hline Diámetro tubería $(\mathrm{m})$ & 0,24 \\
Cc & 0,40 \\
Angulo & 90,0 \\
$\mathrm{~K}$ & 0,40 \\
Pérdida por cambio dirección $\left(\mathrm{seg}^{2} / \mathrm{m}^{5}\right)$ & 9,97 \\
Porcentaje del total $(\%)$ & 0,29 \\
\hline
\end{tabular}

\section{Pérdidas de carga por salida de la tubería (Pst)}

Cuando el agua sale de la tubería de conducción se presentan nuevamente pérdidas debido al cambio de diámetro o por dispositivos colocados al final. La expresión de pérdidas a la salida de la tubería se calcula por medio de la siguiente fórmula.

$$
P_{s t}=\left(\frac{K}{D^{4}}\right) \cdot\left(\frac{8}{\pi^{2} \cdot g}\right) \cdot Q^{2}
$$

En este caso el factor K se le asigna un valor de 1 ya que el área de salida es igual al área de la tubería y cuando esta relación es igual a 1 entonces $\mathrm{K}$ tiene dicho valor (Sotelo, 1993; p. 313). Los valores de los parámetros involucrados se muestran en el cuadro 8, así como los resultados obtenidos, notándose valores relativamente bajos por pérdidas de carga.

\section{Cuadro 8}

Pérdidas de carga por salida de tubería

\begin{tabular}{lc}
\hline Parámetro & Valor (m) \\
\hline $\mathrm{K}$ & 1,00 \\
Diámetro tubería (m) & 0,24 \\
Pérdidas por salida de tubería $\left(\mathrm{seg}^{2} / \mathrm{m}^{5}\right)$ & 24,93 \\
Porcentaje del total $(\%)$ & 0,73 \\
\hline
\end{tabular}

\section{Pérdidas totales de carga}

Las pérdidas totales de carga se pueden obtener de la sumatoria de las pérdidas de carga individuales. Es decir PTC $=$ Per $+\mathrm{Pdr}+$ Petc $+\mathrm{Pdt}+\mathrm{Pcd}+\mathrm{Pst}$. Dichas pérdidas totales alcanzan un valor de $3392,29 \mathrm{seg}^{2} / \mathrm{m}^{5}$ 


\section{Cálculo de caudal en los pozos de bombeo}

Ahora se procede a aplicar la fórmula de abatimiento de cada pozo dispuestas en forma de matriz, cuya incógnita es el caudal de explotación y se debe resolver simultáneamente. Se obtiene una matriz con un número de expresiones igual al número de pozos, que en este caso será de seis, según el número obtenido con la metodología del gran pozo. Si fuera del caso se podría asignar un número arbitrario de pozos y calcular el caudal de cada uno de ellos. La expresión de partida está compuesta por dos términos (E12), uno de ellos corresponde al abatimiento provocado por las pérdidas en el acuífero y el segundo término corresponde a las pérdidas debido a la estructura o armado del pozo $\left(\mathrm{CQ}^{2}\right)$.

$$
s_{i}=\left(\frac{1}{2 \cdot \pi \cdot T}\right) \cdot \sum\left(Q_{i} \cdot \operatorname{Ln}\left\{\frac{R}{r_{i}}\right\}\right)+C Q^{2}
$$

El valor de C utilizado es de 3392,3 $\mathrm{seg}^{2} / \mathrm{m}^{5}$, una transmisividad de $605 \mathrm{~m}^{2} / \mathrm{d}$, un coeficiente de almacenamiento de $6,0 \times 10^{-4}$ y un tiempo de bombeo de 3,6 días que se requiere para calcular el radio de influencia. Las distancias consideradas entre cada pozo de bombeo se indican en el cuadro 9, la distancia del pozo al mismo pozo es considerado como el radio del pozo.

Cuadro 9

Distancia y radio de influencia de la batería de pozos

\begin{tabular}{lc}
\hline Parámetro & Valor (m) \\
\hline Radio influencia & 2857,88 \\
Abatimiento requerido & 19,0 \\
Radio pozo & 0,127 \\
Distancia p1 a p2 & 38,77 \\
Distancia p1 a p3 & 65,30 \\
Distancia p1 a p4 & 124,49 \\
Distancia p1 a p5 & 104,08 \\
Distancia p1 a p6 & 97,90 \\
Distancia p2 a p3 & 26,53 \\
Distancia p2 a p4 & 104,08 \\
Distancia p2 a p5 & 97,90 \\
Distancia p2 a p6 & 104,08 \\
Distancia p3 a p4 & 97,90 \\
Distancia p3 a p5 & 102,00 \\
Distancia p3 a p6 & 118,37 \\
Distancia p4 a p5 & 38,70 \\
Distancia p4 a p6 & 79,59 \\
Distancia p5 a p6 & 38,70 \\
\hline
\end{tabular}

Desenvolviendo la expresión arriba señalada (E12), e incluyendo los valores de cada término conocido se obtiene el conjunto de expresiones que es necesario resolver. La solución de este sistema de ecuaciones debe realizarse de manera simultánea, para lo cual se ha utilizado el método SOLVER, que consiste en un algoritmo de aproximación que permite encontrar la solución por medio de prueba y error en un tiempo relativamente corto.

En las ecuaciones se utilizan valores que se encuentran representados por letras griegas: $\alpha=2,6 \times 10^{-4} ; \psi=2857,9 ; \beta=0,1 ; \delta=38,8 ; \varphi$ $=65,3 ; \gamma=124,5 ; \eta=104,1 ; \lambda=97,9 ; \mu=26,5$ $v=102,0 ; \rho=118,4 ; \sigma=79,6$.

$$
\begin{aligned}
& s_{1}=\alpha\left(Q_{1} \operatorname{Ln} \frac{\Psi}{\beta}+Q_{2} \operatorname{Ln} \frac{\Psi}{\delta}+Q_{3} \operatorname{Ln} \frac{\Psi}{\varphi}+Q_{4} \operatorname{Ln} \frac{\Psi}{\gamma}+Q_{5} \operatorname{Ln} \frac{\Psi}{\eta}+Q_{6} \operatorname{Ln} \frac{\Psi}{\lambda}\right) \\
& s_{2}=\alpha\left(Q_{1} \operatorname{Ln} \frac{\Psi}{\delta}+Q_{2} \operatorname{Ln} \frac{\Psi}{\beta}+Q_{3} \operatorname{Ln} \frac{\Psi}{\mu}+Q_{4} \operatorname{Ln} \frac{\Psi}{\eta}+Q_{5} \operatorname{Ln} \frac{\Psi}{\lambda}+Q_{6} \operatorname{Ln} \frac{\Psi}{\eta}\right) \\
& s_{3}=\alpha\left(Q_{1} \operatorname{Ln} \frac{\Psi}{\varphi}+Q_{2} \operatorname{Ln} \frac{\Psi}{\mu}+Q_{3} \operatorname{Ln} \frac{\Psi}{\beta}+Q_{4} \operatorname{Ln} \frac{\Psi}{\lambda}+Q_{5} \operatorname{Ln} \frac{\Psi}{v}+Q_{6} \operatorname{Ln} \frac{\Psi}{\rho}\right) \\
& s_{4}=\alpha\left(Q_{1} \operatorname{Ln} \frac{\Psi}{\gamma}+Q_{2} \operatorname{Ln} \frac{\Psi}{\eta}+Q_{3} \operatorname{Ln} \frac{\Psi}{\lambda}+Q_{4} \operatorname{Ln} \frac{\Psi}{\beta}+Q_{5} \operatorname{Ln} \frac{\Psi}{\delta}+Q_{6} \operatorname{Ln} \frac{\Psi}{\sigma}\right) \\
& s_{5}=\alpha\left(Q_{1} \operatorname{Ln} \frac{\Psi}{\eta}+Q_{2} \operatorname{Ln} \frac{\Psi}{\lambda}+Q_{3} \operatorname{Ln} \frac{\Psi}{v}+Q_{4} \operatorname{Ln} \frac{\Psi}{\delta}+Q_{5} \operatorname{Ln} \frac{\Psi}{\beta}+Q_{6} \operatorname{Ln} \frac{\Psi}{\delta}\right) \\
& s_{6}=\alpha\left(Q_{1} \operatorname{Ln} \frac{\Psi}{\lambda}+Q_{2} \operatorname{Ln} \frac{\Psi}{\eta}+Q_{3} \operatorname{Ln} \frac{\Psi}{\rho}+Q_{4} \operatorname{Ln} \frac{\Psi}{\sigma}+Q_{5} \operatorname{Ln} \frac{\Psi}{\delta}+Q_{6} \operatorname{Ln} \frac{\Psi}{\beta}\right)
\end{aligned}
$$

La solución de este sistema da como resultado caudales, que varían de 24,6 a 27,0 1/s, para un total de 156 1/s (Cuadro 10). Los caudales se aproximan en gran medida con la realidad, ya que en promedio se extraen $30 \mathrm{l} / \mathrm{s}$ de cada pozo ubicado en la bermas del vertedero principal. Es posible además, por medio de la expresión siguiente calcular el abatimiento en un punto " $\mathrm{X}$ " cercano a la excavación. En esta expresión no se incluyen las pérdidas de carga dentro del pozo

$$
s_{x}=\left(\frac{1}{4 \cdot \pi \cdot T}\right) \cdot \sum\left(Q_{i} \operatorname{Ln} \frac{2,25 \cdot T \cdot t}{r^{2} \cdot S}\right)
$$

Para aplicar esta fórmula y calcular el abatimiento en el punto " $\mathrm{X}$ " que se ubica a las 
Cuadro 10

Caudales calculados para cada pozo

\begin{tabular}{ccc}
\hline Pozo & Caudal $\left(\mathrm{m}^{3} / \mathrm{s}\right)$ & Caudal $(1 / \mathrm{s})$ \\
\hline Pozo 1 & 2305,9 & 26,7 \\
Pozo 2 & 2124,7 & 24,6 \\
Pozo 3 & 2237,2 & 25,9 \\
Pozo 4 & 2331,0 & 27,0 \\
Pozo 5 & 2173,5 & 25,2 \\
Pozo 6 & 2322,0 & 26,9 \\
\hline TOTAL & 13494,3 & 156,2 \\
\hline
\end{tabular}

distancias indicadas en el cuadro 2, se ha escogido el mismo punto que en la metodología anterior y se ha obtenido un valor de descenso de 11,98 m, idéntico que para el procedimiento anterior.

\section{CONCLUSIONES}

Se ha demostrado que es factible por medio de dos métodos analíticos diseñar una batería de pozos alrededor de una excavación para disminuir la presión del agua subterránea, los cuales tienen la ventaja de que se pueden manejar por medio de hojas electrónicas, y constituyen alternativas de análisis de abatimientos de bajo costo en sitios de excavaciones.

Por medio del método del gran pozo de bombeo se ha estimado que son necesarios 6 pozos para obtener un abatimiento de $17 \mathrm{~m}$ en el área del vertedero principal del proyecto hidroeléctrico Angostura. Por su parte con el método de las pérdidas de carga, se ha logrado estimar el caudal de extracción de cada pozo requerido en la excavación del vertedero de una manera más precisa. Dicho análisis se ha realizado considerando la batería de pozos alrededor de la excavación y con una profundidad promedio de 75 metros en cada pozo.
En primera instancia parece que ambos métodos son complementarios; sin embargo, se pueden utilizar de manera independiente. Estos métodos no consideran variaciones de los parámetros hidrodinámicos del acuífero y por lo tanto se deben usar en zonas homogéneas e isotrópicas.

La metodología del gran pozo genera caudales de bombeo mayores que los calculados por la metodología de los caudales. Esto se debe a que se requiere un bombeo mayor para obtener un abatimiento provocado solamente por el acuífero. En el método de los caudales el abatimiento resulta de la fricción tanto en el acuífero como en la estructura del pozo.

Por otro lado se ha encontrado que las mayores pérdidas de carga ocurren dentro de la rejilla del pozo, lo que permite señalar la importancia del diseño geomecánico adecuado a partir de la elección de la rejilla.

\section{REFERENCIAS}

CUSTODIO, E. \& LLAMAS, R., 1983: Hidrología Subterránea. Tomo I. -1157 pp. Ed. Omega. Barcelona.

DRISCOLL, F., 1986: Groundwater and Wells. -1089 pp. Johnson. Minnesota.

HERTH, W. \& ARNDTS, E., 1994: Theorie und Praxis der Grundwasserabsenkung. - 357 págs. Ed. Ernst \& Sohn, Berlin.

SOTELO, G., 1993: Hidráulica General (19va. ed.). -561 p. Ed. Limusa, México. D.F.

VARGAS, A., 1997: Hydrogeologische und geohydraulische Untersuchungen im Tal des Río Reventazón, Costa Rica. - Giessener Geologische Schriften, 61: 1-189.

VARGAS, A. \& CERVANTES, F., 2000: Caracterización de acuíferos en el área de la presa del proyecto hidroeléctrico Angostura, Turrialba, Costa Rica. - Rev. Geól. América Central, 23: 65-76. 